\section{Case studies on implant removal influencing the resolution of inferior alveolar nerve injury}

N. Khawaja ${ }^{1}$ and T. Renton ${ }^{2}$
IN BRIEF

- Outlines types of nerve injury including those that might result from implant placement.

- Describes techniques for assessing implant related sensory disturbance and recovery.

- Recommends best management of suspected or actual implant related nerve injuries and treatment algorithm.

\begin{abstract}
Introduction Nerve injury during implant placement is a preventable, serious complication with major medico-legal implications. The incidence of implant related inferior alveolar nerve (IAN) injuries varies from 0-40\%. This article presents four cases of IAN injury following mandibular implant placement with early removal, referred to the oral surgery department, King's College Hospital, London. Objectives To assess sensory disturbance and recovery in patients with implant related IAN injury and assess whether early removal of the implants promotes neural recovery. To present recommendations on how to best deal with suspected/actual nerve injuries and prevent their occurrence. Methods Over the last two years, four patients referred for specialist opinion to the oral surgery department were identified as having sustained implant related IAN injury with early removal (18 hours to four days post-injury). Data were collected by referral to health records. Results All patients suffered from numbness of the affected inferior alveolar dermatome with three out of four cases also experiencing some form of neuropathic pain. Cases 1 and 2, who had their implants removed at 18 and 36 hours post-injury respectively, regained almost complete sensory recovery. Case 1 also had adjunctive NSAID and steroid therapy. Cases 3 and 4 suffered complete numbness in the inferior alveolar dermatome and did not experience any improvement in sensation following removal of their implants at two and four days post-injury respectively. Both patients with significant persistent neuropathy (Cases 3 \& 4 ) reported functional problems. Conclusion This cohort of patients may demonstrate that early removal of implants associated with IAN injury (less than 36 hours post-injury) may assist in minimising or even resolution of IAN neuropathy. Adjunctive corticosteroid and high dose non-steroidal anti-inflammatory therapy also appears effective. Implant related IAN injury is a preventable, elective procedure and a suggested protocol of management of suspected injuries, for dental practitioners, is presented.
\end{abstract}

\section{INTRODUCTION}

\section{Pain and functional problems}

The incidence of implant related nerve injuries varies from $0-40 \% . .^{5-12}$ Injuries to the inferior alveolar nerve during dental surgery remains a common and complex clinical problem with major medicolegal implications. ${ }^{.}$Sensory nerve injury often results not in numbness which is a common misapprehension but in altered sensation and/or pain. This commonly

\footnotetext{
${ }^{1 *}$ Walport Academic Clinical Fellow Oral Surgery,

${ }^{2}$ Professor of Oral Surgery, Department of Oral Surgery, King's College London Dental Institute, Denmark Hill

Campus, Bessemer Rd, London, SE5 9RS

${ }^{*}$ Correspondence to: Dr Nadine Khawaja

Email: nadine.khawaja@kcl.ac.uk
}

\section{Refereed Paper}

Accepted 6 February 2009

DOI: $10.1038 /$ sj.bdj.2009.258

${ }^{\circledR}$ British Dental Journal 2009; 206: 365-370 interferes with speech, eating, kissing, make-up application, shaving and drinking, in fact, just about every social interaction we take for granted. ${ }^{2}$ Following oral implant rehabilitation, the patient usually expects and experiences significant improvements, not only regarding jaw function, but also in relation to dental, facial, and even overall body image. ${ }^{3}$ Thus these injuries have a significant negative effect on the patient's quality of life and the iatrogenesis of these injuries compounds the negative psychological effects for these injuries. ${ }^{4}$

\section{Type of nerve injury}

Sensory nerve injuries may be caused by mechanical, chemical, thermal stimuli and ischaemia. Several modalities of nerve injury including direct trauma, inflammation and infection are the main causes of post-operative neural disturbances. ${ }^{22}$
These injuries may occur during preparation or placement of an implant. They may be directly related to the depth of preparation, implant length or width. ${ }^{13}$ Trauma may be direct (mechanical or chemical) or indirect (haemorrhage or scarring). Alkalinic nerve injuries from irrigation of the implant bed with sodium hypochlorite (not recommended practice) solutions should be avoided and saline used instead.

Trauma to a peripheral nerve may result in a deficiency ranging from total loss of sensation (anaesthesia) to a mild decrease in feeling (mild hypoesthesia). Troublesome altered sensation (dysesthesia) or pain, both of which may be constant or spontaneous or evoked (Allodynia is pain response to normal stimulus). The pathophysiology of these IAN neuropathies is complex, and treatment results are often disappointing. Many authors provide unrealistic expectations 
for early microsurgical repair and the presence of anaesthesia, dysesthesia or spontaneous pain also indicates poor prospects for recovery even with surgical intervention. ${ }^{2,11}$ Overall, 25\% of patients with iatrogenic paraesthesia suffer permanent effects. ${ }^{28,29}$

Based on studies evaluating the recovery of lingual nerve injuries subsequent to third molar surgery, Blackburn ${ }^{26}$ and Mason $^{27}$ report that $90 \%$ of lingual nerve injuries are temporary and resolve at 8-10 weeks post-surgically. The lingual nerve is not comparable with the IAN in that it lies in soft tissue, is not confined within a bony canal as is the IAN, thus it will be less prone to ischaemic injury in relation to primary or secondary haemorrhage. Thus we must be careful of making incorrect assumptions regarding the potential for recovery of IAN injuries.

It is worth noting that the known method of causing maximum neuropathic pain in the rat model is to ligate the sciatic nerve. A similar effect may occur within the IAN bony canal after haemorrhage or debris competes with the nerve for space. This may explain the many patients suffering with neuropathic pain associated with IAN iatrogenic nerve injuries.

There are rare reports of resolution of implant related IAN neuropathies at over four years but these do not comply with normal reports of peripheral sensory nerve injuries. ${ }^{23-25}$

\section{Current management}

There are currently no standardised guidelines on the management of implant related nerve injury. In contrast to other surgical specialties who surgically explore all known peripheral sensory nerve injuries (plastics and orthopaedics) immediately, we, as a profession, sit and wait for these sensory nerve injuries to resolve.

Many authors recommend referral of injuries before four months but this may be too late for many peripheral sensory nerve injuries. ${ }^{11}$ We now understand that after three months, permanent central and peripheral changes occur within the nervous system subsequent to injury, that are unlikely to respond to surgical intervention. ${ }^{2}$

Hegedus and Diecidue ${ }^{11}$ recommend removing the implant immediately and replacing it several days later when initial healing has taken place allowing optimal neural healing.

\section{Planning}

Many injuries can be prevented through better patient selection, planning and execution of procedures. ${ }^{13-19}$ Patient management can often be improved by informed consent and post-operative care with early referral for nerve injuries. Many practitioners use software to assist in the planning of implants and for the identification of the IAN canal position with the specific aim to place the implants more than $2 \mathrm{~mm}$ from the IAN canal. ${ }^{14}$ It may be prudent to highlight that it is the practitioner that draws in the IAN canal for assessment which will not be objective and is subject to error. More recently Abarca et al. ${ }^{4}$ have highlighted the necessity for cross-sectional imaging even for surgical procedures in the symphyseal region due to unforeseen nerve injuries. Several papers have reported the mental nerve anatomy. ${ }^{14-16}$ These suggest leaving a $2 \mathrm{~mm}$ zone of safety between an implant and the coronal aspect of the nerve on panoramic and periapical films prior to implant placement; use of CT (cone beam CT) scans when these techniques often do not provide clarity with respect to the position of the nerve; surgical corroboration of the mental nerve's position when an anterior loop of the mental foramen is suspected of being present or if the zone of safety (in millimetres) for implant placement is clear. Clinicians must have an awareness that certain prep drills are up to $1.5 \mathrm{~mm}$ longer than the placed implant. In general, altered lip sensations are preventable if the nerve and mental nerve is radiographically located and this knowledge is employed when performing surgical procedures in the foraminal area. ${ }^{14}$

Avoidance of nerve injury is sometimes attempted by using techniques including inferior alveolar nerve lateralisation and posterior alveolar distraction, however, these high-risk procedures are more likely to result in inferior alveolar nerve defect regardless of the surgeon's experience. ${ }^{10,17}$ Both the lingual and inferior alveolar nerves are at risk of injury during the administration of an inferior alveolar nerve block, but are very rare (approximately 1:20-30,000).18-21 Bone

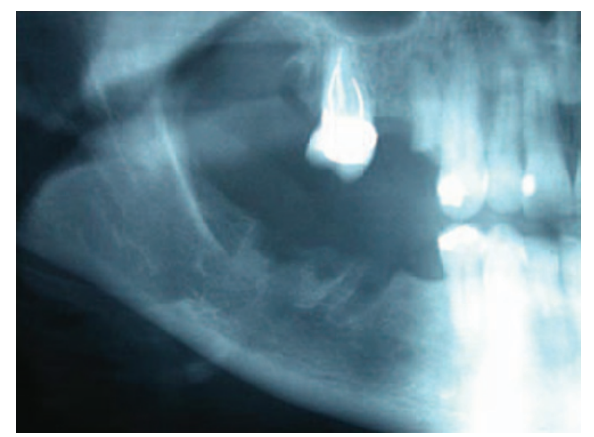

Fig. 1 Case 1, two implants removed

harvesting close to the mental nerve or in the retromolar region may also cause nerve injuries. ${ }^{21}$

\section{Informed consent}

All patients must have realistic expectations and also be warned of IAN injury. Assessment of risk must be undertaken in order to appropriately advise the patient with regard to alternative treatment plans and include this possibility in the consent forms. ${ }^{22}$ The information should be explicit with ensuring that the patient is aware that nerve injury may cause altered sensation (numbness, pain or troublesome altered sensation) that may be intermittent or constant, temporary or permanent. The patient must also be warned that the neuropathic area may affect all of the IAN dermatome extra- and intra-orally (whole of skin and vermillion of lip and chin on each side and all lower quadrant teeth and associated buccal gums) or just part of the area. When inferior alveolar nerve injury occurs it is imperative that the surgeon recognise the injury and advise the patient appropriately.

The most desirable outcome after nerve injury is spontaneous return of normal sensation. The likelihood of this occurring depends on both the severity of the injury and the nerve involved. The outcome of nerve injury will depend on multiple factors including: case selection, planning and assessment of the related anatomy, informed consent, surgical expertise and execution.

This article presents four cases of IAN injury following mandibular implant placement with early removal referred to the oral surgery department in King's College Hospital, London. As per standard technique, all implants were removed by reattaching the driver to the head of 
Table 1 Neurosensory function tests

\begin{tabular}{|c|c|c|c|c|c|c|c|c|c|}
\hline $\begin{array}{l}\text { Duration } \\
\text { post injury }\end{array}$ & $\begin{array}{l}\text { Neuropathic } \\
\text { area }\end{array}$ & $\begin{array}{l}\% \text { skin/ vermil- } \\
\text { lion affected }\end{array}$ & LTPS & SBD & TPDT & MTPDT & $\begin{array}{l}\text { SF skin/ } \\
\text { vermillion }\end{array}$ & $\begin{array}{l}\text { Neuropathic pain/ } \\
\text { neuralgia }\end{array}$ & $\begin{array}{l}\text { Other findings } \\
\text { Function }\end{array}$ \\
\hline $\begin{array}{l}\text { Case } 1 \\
1 \text { day }\end{array}$ & Right IAN & $\begin{array}{l}25-30 \% / \\
60 \%\end{array}$ & $\checkmark$ & $\checkmark$ & $\uparrow$ & $\checkmark$ & $3-4 / 6-7$ & - & $\begin{array}{l}\text { Electric-shock when } \\
\text { implant removed }\end{array}$ \\
\hline $\begin{array}{l}\text { Case1 } \\
6 \text { weeks }\end{array}$ & Right IAN & $10 \% / 10 \%$ & $\checkmark$ & $\checkmark$ & - & $\downarrow$ & $7 / 10$ & $\begin{array}{l}\text { Cold and mechanical } \\
\text { allodynia }\end{array}$ & $\begin{array}{l}\text { Marked reduction in neu- } \\
\text { ropathic area over } 2 / 12\end{array}$ \\
\hline $\begin{array}{l}\text { Case } 2 \\
7 \text { days }\end{array}$ & Left IAN & Pt report $100 \%$ & - & - & - & - & - & - & - \\
\hline $\begin{array}{l}\text { Case } 2 \\
3 \text { months }\end{array}$ & Left IAN & $1 \% / 0 \%$ & $\checkmark$ & $\checkmark$ & $\checkmark$ & $\checkmark$ & $10 / 10$ & - & Nil functional problems \\
\hline $\begin{array}{l}\text { Case } 3 \\
7 \text { days }\end{array}$ & Right IAN & $100 \% / 100 \%$ & - & - & - & - & - & - & Dribbling and lip biting \\
\hline $\begin{array}{l}\text { Case } 3 \\
2 \text { months }\end{array}$ & Right IAN & $100 \% / 100 \%$ & $\downarrow$ & $\downarrow$ & $\downarrow$ & $\checkmark$ & $14 / 11$ & $\begin{array}{l}\text { Mechanical allodynia } \\
\text { with hyperalgesia }\end{array}$ & $\begin{array}{l}\text { Difficulty eating drinking } \\
\text { speaking sleeping }\end{array}$ \\
\hline $\begin{array}{l}\text { Case } 4 \\
2 \text { days }\end{array}$ & Left IAN & $\begin{array}{l}100 \% / \\
100 \%\end{array}$ & $\downarrow$ & $\downarrow$ & $\downarrow$ & $\begin{array}{l}\checkmark \\
\text { pain }\end{array}$ & - & - & - \\
\hline $\begin{array}{l}\text { Case } 4 \\
3 \text { months }\end{array}$ & Left IAN & $\begin{array}{l}100 \% / \\
100 \%\end{array}$ & $\downarrow$ & $\downarrow$ & $\downarrow$ & $\checkmark$ & $14 / 11$ & $\begin{array}{l}\text { Mechanical allodynia } \\
\text { with hyperalgesia }\end{array}$ & $\begin{array}{l}\text { Nil scarring or functional } \\
\text { deficits }\end{array}$ \\
\hline
\end{tabular}

the implant and using the handpiece in reverse.

\section{CASE 1}

\section{(55-year-old female with implant removal at 18 hours)}

\section{Implant placement}

Two implants were placed in the lower right first molar region edentulous free end saddle (Fig. 1). The patient was telephoned on the evening of surgery by the practitioner and she reported persistent numbness of her lower right lip and chin.

\section{Implant removal}

The patient returned for removal of both implants the following morning, approximately 17.5 hours post-surgery). During removal of the implants, the patient described an electric-shock feeling, implying some neural continuity.

She was prescribed high dose Ibuprofen (800 mg TDS), amoxicillin $500 \mathrm{mg}$ TDS 5/7 and prednisolone 50 $\mathrm{mg}$, once daily for the next five days with step-down $10 \mathrm{mg}$ over the ensuing five days.

\section{Neurosensory function tests (Table 1)}

She was urgently referred to the oral surgery department and King's College Hospital for further assessment the day following surgery. Dental panoramic tomography (DPT) showed that the implant sockets were prepared close to IAN canal but did not transect it.
Objective measures of area of neuropathy ranged from $25-30 \%$ of the extra-oral region and around 60\% of the intra-oral region from the lower right first molar forwards with reduced mechanical sensory function along with light touch, sharp blunt discrimination, moving point discrimination and twopoint discrimination.

The patient's subjective function was reduced at 3 to 4 (out of a possible 10) on the dermal area of the neuropathic region of the right inferior alveolar nerve and 6 to 7 on the vermillion region.

She presented with a minimal area of paraesthesia over the vermillion of the right neuropathic region of the right IAN but no background burning pain.

\section{Review}

On review in the oral surgery clinic, six weeks following injury, the patient presented with markedly improved sensation. A small region of the chin supplied by the right IAN $(<5 \%$ of possible area) had persistent neurosensory deficit with some element of cold/mechanical allodynia.

The patient is on a three-month review of her resolving neurosensory deficit.

\section{CASE 2}

\section{(56-year-old female with implant removal at 24-36 hours)}

\section{Implant placement}

This patient was undergoing implant

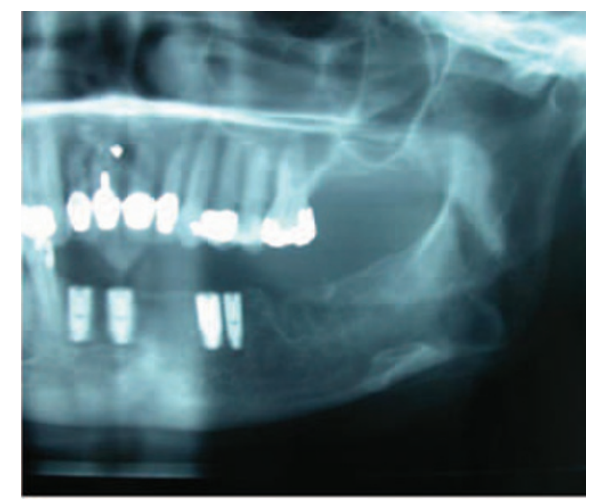

Fig. 2 Case 2, radiograph

placement in the lower right and left quadrants for a lower implant retained partial denture. The patient was contacted the night of implant placement in the lower left first molar region and reported improvement in sensation but complained of some discomfort around the area of the implant.

Overnight the patient experienced increased numbness of the whole of her lower left lip, chin and lower anterior teeth.

\section{Implant removal}

She presented to the practitioner the morning following surgery with significant numbness in the area of the inferior alveolar nerve. The implant was then removed by the practitioner at the same visit approximately 24 hours following implant placement (Fig. 2). No medication was prescribed to the patient and she was not taking any regular medication/analgesics. 


\section{Neurosensory function tests (Table 1)}

The patient presented to the oral surgery clinic two months following injury with markedly improved sensation. DPT examination demonstrates the implants prepared close to the inferior alveolar canal but did not transect it.

On examination, she had a residual minimal area of left inferior alveolar nerve neuropathy with less than $1 \%$ of the possible dermatome just affecting less than $1 \mathrm{~cm}$ width of the vermillion on the lower left aspect of the lip. She did not experience any spontaneous/ evoked pain, or functional problems.

\section{Review}

The patient did not wish to be reviewed for her resolving neurosensory deficit.

\section{CASE 3}

\section{(46-year-old female with} implant removal at two days)

\section{Implant placement}

Following implant placement in the lower left second premolar region, the patient experienced persistent numbness of left lower lip and chin after local anaesthetic wore off.

\section{Implant removal}

The implant was removed two days after surgery (Fig. 3). No medication was prescribed to the patient and she was not taking any regular medication/analgesics.

\section{Neurosensory function tests (Table 1)}

Three months following injury the patient presented to the oral surgery department, February 2007, with significant altered sensation of the left inferior alveolar region. She was experiencing severe evoked pain on cold or tactile stimulation over the region. Functionally the patient had significant problems with eating, speaking, drinking, kissing and sleeping. There was no improvement in sensation over the three month period.

DPT demonstrates implant preparation in close proximity to the mental nerve foramen. On examination, the area of neuropathy involved $100 \%$ of the inferior alveolar nerve dermatome extra-orally with a degree of thermal and mechanical allodynia with hyperalgesia.
Intra-orally, she had a significant neuropathic area affecting the mental nerve division from the lower left second premolar forwards with extreme difficulty in brushing her teeth. Mechanosensory function was reduced and subjective function was elevated out of a 'normal sensation' of 10 ranging from 11-14 in line with mechanical allodynia.

\section{Review}

The patient is no longer under review. She accepts her permanent neuropathy and is managing her evoked pain with topical lidocaine patches.

\section{CASE 4}

\section{(39-year-old female with implant removal at four days)}

\section{Implant placement}

Following implant placement in the lower right second premolar region the patient experienced persistent numbness of the right lower lip and chin after the local anaesthetic wore off.

\section{Implant removal}

The implant was removed four days after surgery (Fig. 4). No medication was prescribed to the patient and she was not taking any regular medication/analgesics.

\section{Neurosensory function tests (Table 1)}

Two months following injury the patient presented to the oral surgery department, October 2006, with significant altered sensation of the right inferior alveolar region. She did not experience any spontaneous/evoked pain, or functional problems. There was no improvement in sensation over the two month period.

Right sectional DPT demonstrates bone defect of the implant preparation sitting millimetres above the mental nerve foramen.

On examination, the area of neuropathy involved $100 \%$ of the inferior alveolar nerve dermatome with a degree of mechanical allodynia with hyperalgesia.

Intra-orally, she had a significant neuropathic area affecting the mental nerve division from the lower right second premolar forwards. Subjective function was elevated out of a 'normal sensation' of 10 ranging from 11-14, inline with mechanical allodynia.

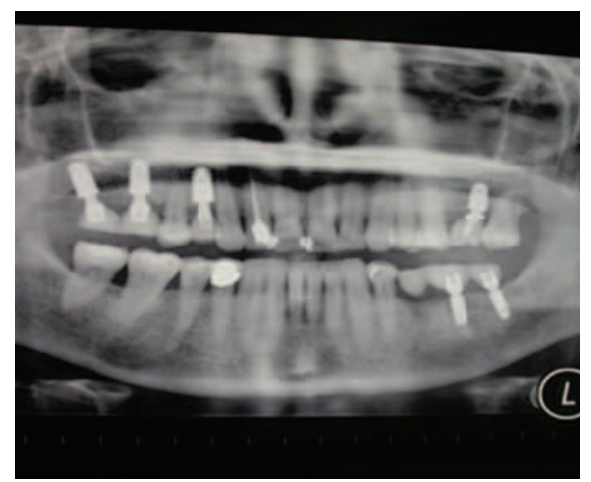

Fig. 3 Case 3, OPG

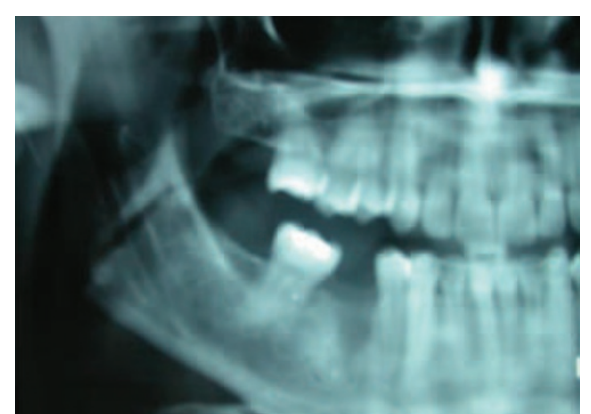

Fig. 4 Case 4, radiograph

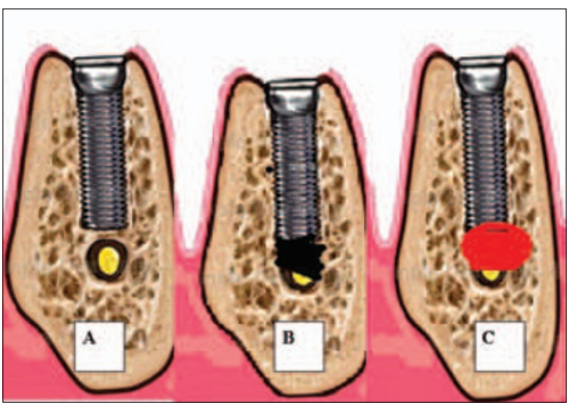

Fig. 5 Proximity of the implants to the IAN canal

\section{Review}

The patient is no longer under review and accepts her permanent neuropathy.

\section{OBSERVATIONS}

All patients suffered from numbness of the affected inferior alveolar dermatome with all cases also experiencing some form of neuropathic pain.

The two patients (Cases 1 and 2) who had their implants removed within 36 hours post-surgery regained almost complete sensory recovery. Cases 3 and 4 suffered complete numbness in the inferior alveolar dermatome and did not experience any improvement in sensation following removal of their implants at two and four days respectively. Case 1 also had adjunctive high dose nonsteroidal-anti-inflammatories (NSAIDs) and steroid therapy which may in theory 
assist with minimising neural inflammation. Cases 2, 3 and 4 were not prescribed any adjunctive medication.

\section{DISCUSSION}

All four cases suffered from neuropathic pain in this cohort. This may suggest that the type of injury caused by implant placement is due to primary or secondary ischaemia of the IAN by haemorrhage into the canal, rather than direct mechanical trauma by the implant itself. 'Cracking' of the IAN canal roof by its close proximity to preparation of the implant bed (millimetres) may cause haemorrhage into the canal or deposition of debris which may compress and cause ischaemia of the nerve. This constrictive effect on the nerve may persist if the implant is left in situ, even if the implant is 'backed-up' or a shorter implant is placed (Fig. 5).

Intraoperatively risk factors may also include a sudden give during preparation (penetration of canal wall as reported in two of four cases) or a reported electricshock type feeling during preparation (one of four cases). If there is an inferior alveolar arterial or venous bleed (two cases presented) it may be advisable not to place the implant and to wait two to three days to ensure no nerve damage has occurred and then place the implant in granulation tissue which should not compromise the success of the implant. However, there is no evidence to support this practice yet.

Two of the four cases who had their implants removed within 36 hours experienced a dramatic improvement in sensation, although there is no way of knowing whether the injuries would have resolved if the implants were left in situ. Removal of the implants at two days or more following nerve injury in our cases did not show an improvement in sensation and may place patients at a higher risk of permanent altered sensation. On this basis we suggest that each patient should be contacted after the local anaesthetic has worn off (approximately six hours following the procedure) (Fig. 6). If they report persistent numbness they should be asked to return to the practice for removal of the implant as soon as practicable. The clinician should take a thorough pain/numbness

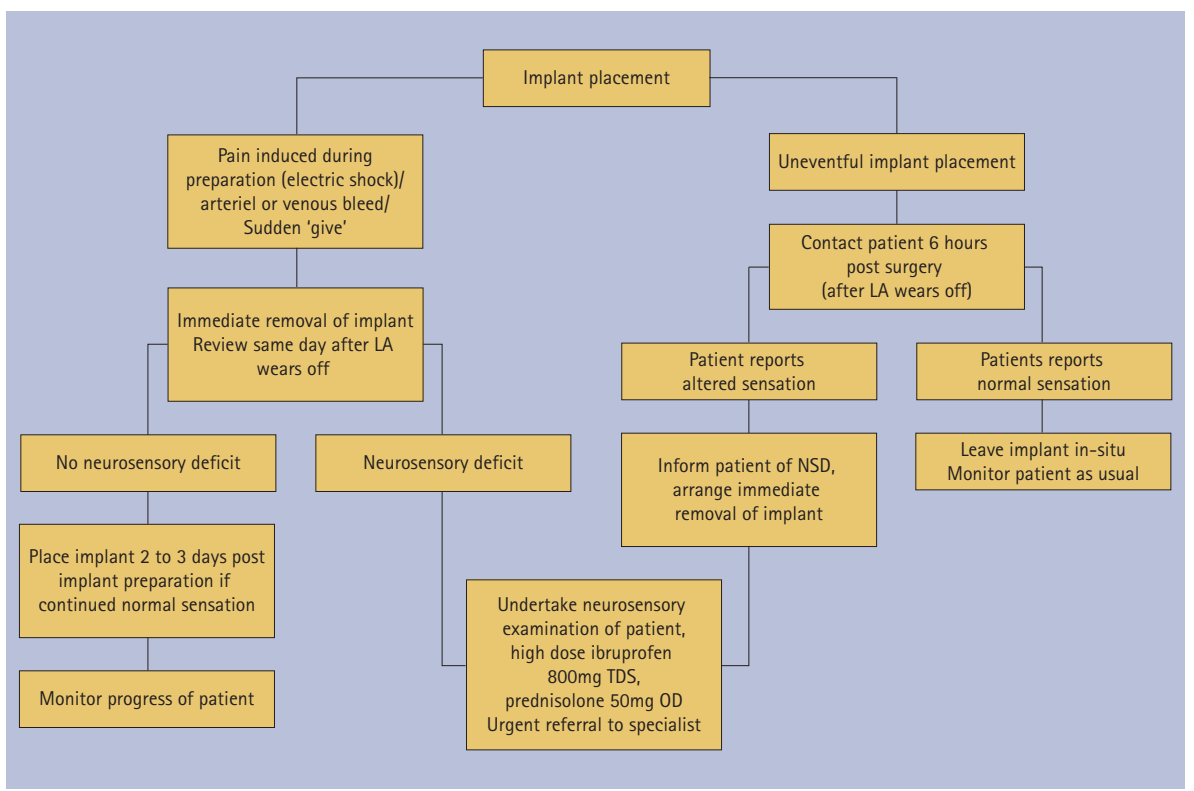

Fig. 6 Flow chart of suggested patient management

\section{Table 2 Objective measures}

\begin{tabular}{|c|c|}
\hline Neuropathic area (NA) & $\begin{array}{l}\text { Lightly touch the lip and chin with a wisp of cotton at the end of a cotton } \\
\text { swab, moving into and away from the neuropathic region, to determine } \\
\text { if and what size neuropathic area is present. This area is then converted } \\
\text { to a percentage of the dermatome affected extra-orally and intra-orally } \\
\text { separately. It may be pertinent to mark and take a photograph of the } \\
\text { neuropathic area for future comparison. }\end{array}$ \\
\hline $\begin{array}{l}\text { Light touch pressure } \\
\text { sensibility (LTPS) }\end{array}$ & $\begin{array}{l}\text { Lightly touch the neuropathic area to determine the perception threshold. } \\
\text { Increase applied pressure until perception of the stimulus is established. } \\
\text { The test is considered positive if the patient can determine light touch } \\
\text { of a cotton wisp. }\end{array}$ \\
\hline Subjective function (SF) & $\begin{array}{l}\text { Repeated application of a blunt probe compare the subjective function on } \\
\text { the lip and chin with the patient's unaffected side (range } 0-10 \text { ). Equal sen- } \\
\text { sation affected and non-affected side }=10 \text { and no feeling on affected side } \\
=0 \text {, increased sensation on affected side (pain-mechanical allodynia) }>10 \text {. }\end{array}$ \\
\hline $\begin{array}{l}\text { Sharp blunt } \\
\text { discrimination (SBD) }\end{array}$ & $\begin{array}{l}\text { Apply a sharp right angle dental probe to the area, with indentation but } \\
\text { with no breach of the mucosa/skin. The patient is then asked to compare } \\
\text { this sensation to that produced by a blunt region of the probe. The test } \\
\text { is considered positive if the patient recognises three out of five of each } \\
\text { stimulus correctly. }\end{array}$ \\
\hline $\begin{array}{l}\text { Two point discrimination } \\
\text { threshold (TPDT) }\end{array}$ & $\begin{array}{l}\text { Apply a pointed calliper (starting } 2 \mathrm{~mm} \text { apart) to the mucosa/skin, without } \\
\text { movement and gradually open to a maximum distance of } 1 \text { centimetre. The } \\
\text { distance where the patient reports feeling two separate points is recorded. }\end{array}$ \\
\hline $\begin{array}{l}\text { Moving two point discrimi- } \\
\text { nation threshold (MTPDT) }\end{array}$ & As for TPDT, but moving the callipers. \\
\hline Neuralgia & $\begin{array}{l}\text { Ask the patient if they experience any spontaneous/ evoked pain } \\
\text { and document. } \\
\text { Apply ethyl chloride and a mirror handle warmed to } 43^{\circ} \mathrm{C} \text { to affected and } \\
\text { unaffected areas to determine if the patient feels normal cold or heat } \\
\text { sensation or pain (thermal allodynia). }\end{array}$ \\
\hline Other findings & $\begin{array}{l}\text { Document any trauma or scarring due to lip biting, any unusual symptoms } \\
\text { during placement or removal of implant, any improvement or change in } \\
\text { sensation following injury and any associated functional problems (eating, } \\
\text { drinking, shaving, speaking and applying make-up). }\end{array}$ \\
\hline
\end{tabular}

history and document nerve function of the patient. Objective measures (Table 2) should be taken as well as asking the patient about any functional problems. Once nerve injury is confirmed, removal of the implant immediately may be prudent as reported in these cases. This may allow faster dispersion of the haemorrhage/debris, allowing improved peripheral nerve regeneration. ${ }^{11}$ We, therefore, 
suggest that arrangements should be made for removal of the implant as soon as nerve injury is suspected (within 36 hours post-surgery).

Adjunctive therapy may be advisable as in Case 1 . Since the altered sensation may be due to an inflammatory reaction, a course of steroid and NSAID treatment may minimise inflammation and reduce damage to the nerve (oral prednisolone 1 mg per kg per day (maximum $80 \mathrm{mg}$ ) for first week with stepping down by $10 \mathrm{mg}$ daily over the following week,) and a high dose of non-steroidal anti-inflammatory medication (such as ibuprofen [800 milligrams] three times per day) should be prescribed for three weeks. There is a minimal evidence base for steroid medication for the prevention of peripheral sensory nerve injuries although it is routinely prescribed for Bell's palsy and viral infections of the facial nerve. ${ }^{17}$ The combination of high-dose steroids and NSAIDs can be associated with significant complications including upper gastrointestinal ulceration if prescribed long term. Even in the short term (two weeks) prescription of these drugs should be undertaken with consideration to the patient's medical history and caution.

\section{CONCLUSION}

IAN injury neuropathy associated with implants is generally considered to be permanent even with implant removal which unfortunately is often undertaken late due to delayed referral. As a result many specialists no longer remove implants in patients presenting with IAN neuropathy (Pogrel personal communication). Prevention of injury is definitely better than cure, however, this cohort of patients may demonstrate that non placement or early removal of implants associated with IAN injury may assist in minimising or even resolution of IAN neuropathy and a suggested protocol is presented (Fig. 6). These recommendations are preliminary in nature and further studies are need to assess the protocol's efficacy. Our oral surgery department will continue to audit the outcome of our implant related nerve injury referrals for whom we undertake this management.

1. Caissie R, Goulet J, Fortin M, Morielle D. latrogenic paresthesia in the third division of the trigeminal nerve: 12 years of clinical experience. J Can Dent Assoc 2005; 71: 185-190.

2. Ziccardi $\vee B$, Assael $L A$. Mechanisms of trigeminal nerve injuries. Atlas Oral Maxillofac Surg Clin North Am 2001; 9: 1-11.

3. Kiyak H A, Beach B H, Worthington P, Taylor T et al. The psychological impact of osseointegrated dental implants. Int J Oral Maxillofac Implants 1990; 5: 61-69

4. Abarca M, van Steenberghe D, Malevez C, De Ridder J, Jacobs R. Neurosensory disturbances after immediate loading of implants in the anterior mandible: an initial questionnaire approach followed by a psychophysical assessment. Clin Oral Investig 2006: 10: 269-277.

5. Delcanho R E. Neuropathic implications of prosthodontic treatment. J Prosthet Dent 1995; 73: 146-152.

6. Rubenstein J E, Taylor T D. Apical nerve transection resulting from implant placement: a 10-year followup report. J Prosthet Dent 1997; 78: 537-541.

7. Wismeijer $D$, van Mass M, Vermeeren J, Kalk W. Patient's perception of sensory disturbances of the mental nerve before and after implant surgery: a prospective study of 110 patients. Br J Oral Maxillofac Surg 1997; 35: 254-259.

8. Bartling $R$, Freeman $K$, Kraut $R$ A. The incidence of altered sensation of the mental nerve after mandibular implant placement. J Oral Maxillofac Surg 1999; 57: 1408-1410.

9. Walton J N. Altered sensation associated with implants in the anterior mandible: a prospective study. J Prosthet Dent 2000; 83: 443-449.

10. Von Arx T, Hafliger J, Chappuis V. Neurosensory disturbances following bone harvesting in the symphysis: a prospective clinical study. Clin Oral Implants Res 2005; 16: 432-439.

11. Hegedus F, Diecidue RJ. Trigeminal nerve injuries after mandibular implant placement - practical knowledge for clinicians. Int J Oral Maxillofac Implants 2006; 21: 111-116.
12. Hillerup $S$, Jensen R. Nerve injury caused by mandibular block analgesia. Int J Oral Maxillofac Surg 2006; 35: 437-443.

13. Worthington P. Injury to the inferior alveolar nerve during implant placement: a formula for protection of the patient and clinician. Int J Oral Maxillofac Implants 2004; 19: 731-734.

14. Greenstein G, Tarnow D. The mental foramen and nerve: clinical and anatomical factors related to dental implant placement: a literature review. J Periodontol 2006; 77: 1933-1943.

15. Shankland W E. Anatomical considerations for mandibular implant surgery. 2. Two common postoperative problems. Dent Today 1991; 10: 48-9, 51

16. Arzouman M J, Otis L, Kipnis V, Levine D. Observations of the anterior loop of the inferior alveolar canal. Int J Oral Maxillofac Implants 1993; 83: 443-449.

17. Von Arx D P, Simpson M T. The effect of dexamethasone on neurapraxia following third molar surgery. Br J Oral Maxillofac Surg 1989; 27: 477-480.

18. Pogrel M A, Thamby S. Permanent nerve involvement resulting from inferior alveolar nerve blocks. J Am Dent Assoc 2000; 131: 901-907.

19. Pogrel M A, Maghen A. The use of autogenous vein grafts for inferior alveolar and lingual nerve reconstruction. J Oral Maxillofac Surg 2001; 59: 985-988.

20. Haas D A, Lennon D. A 21 year retrospective study of reports of paresthesia following local anesthetic administration. J Can Dent Assoc 1995; 61: 319-320, 323-326, 329-330

21. Hillerup S. latrogenic injury to oral branches of the trigeminal nerve: records of 449 cases. Clin Oral Investig 2007: 11: 133-134

22. Nazarian $Y$, Eliav E, Nahlieli O. Nerve injury following implant placement: prevention, diagnosis and treatment modalities. Refuat Hapeh Vehashinayim 2003; 20: 44-50, 101.

23. Elian N, Mitsias M, Eskow R, Jalbout Z N et al. Unexpected return of sensation following 4.5 years of paresthesia: case report. Implant Dent 2005; 14: 364-367.

24. Robinson P P. Observations on the recovery of sensation following inferior alveolar nerve injuries. Br J Oral Maxillofac Surg 1988; 26: 177-189.

25. Robinson P P, Loescher A R, Smith K G. A prospective, quantitative study on the clinical outcome of lingual nerve repair. Br J Oral Maxillofac Surg 2000; 38: 255-263.

26. Blackburn CW. A method of assessment in cases of lingual nerve injury. Br J Oral Maxillofac Surg 1990; 28: 238.

27. Mason D A. Lingual nerve damage following lower third molar surgery. Int J Oral Maxillofac Surg 1988; 17: 290-294.

28. Zuniga J R, Chen N, Phillips C L. Chemosensory and somatosensory regeneration after lingual nerve repair in humans. J Oral Maxillofac Surg 1997; 55: 2-13.

29. LaBanc J P, Van Boven R W. Surgical management of inferior alveolar nerve injuries. Oral Maxillofac Surg Clin North Am 1992; 4: 425-437. 